\title{
Mouse Double Minute 2 Actively Suppresses p53 Activity in Oocytes during Mouse Folliculogenesis
}

\author{
Chen-Xi Zhang, ${ }^{*}$ Qin Zhang, ${ }^{*}$ Y Yin-Yin Xie, ${ }^{*}$ Xue-Yan He, ${ }^{*}$ Cong Xiang, ${ }^{*}$ Xiao-Shuang Hou, ${ }^{*}$ Ying Zhou, ${ }^{*}$ Lai Chen, ${ }^{*}$ \\ Guo-Xin Zhang,* and Geng Liu*
}

From the State Key Laboratory of Pharmaceutical Biotechnology and MOE Key Laboratory of Model Animals for Disease Study,* Model Animal Research Center of Nanjing University, Nanjing; and the Institute of Biophysics, ${ }^{\dagger}$ Chinese Academy of Sciences, Beijing, China

\author{
Accepted for publication \\ September 27, 2016. \\ Address correspondence to \\ Geng Liu, Ph.D., Model Ani- \\ mal Research Center of Nanjing \\ University, 12 Xuefu Road, \\ Pukou High Tech District, \\ Nanjing, JiangSu Province \\ 210061, China. E-mail: \\ liug53@nju.edu.cn.
}

\begin{abstract}
The $p 53$ signaling network is indispensible in cellular stress responses and tumor suppression. Negative regulations of p53 by mouse double minute 2 (MDM2) and its homolog MDM4 are an integrated component of the network and have been implicated in regulating the stress responses and the maintenance of normal development and homeostasis of multiple somatic cell lineages. However, the regulatory role of MDM2 on p53 and stress responses in female germ cells remains undetermined. Here, we used the Cre-loxP system to delete $M d m 2$ in oocytes at different stages of folliculogenesis in mice. Mdm2 deletion resulted in a clear p53 nuclear accumulation in the oocytes and impeded fertilities with early follicular loss in mice, resembling human premature ovarian failure phenotypes. These phenotypes were fully rescued by concurrent deletion of $p 53$ in mice. In addition, Nutlin-3, a small molecule compound that inhibited the binding of MDM2 to p53, also promoted p53-dependent oocyte death. Although cancer therapeutic agents 5-fluorouracil and doxorubicin could not induce a robust p53 activation in the wild-type oocytes, they induced p53 nuclear accumulation in the Mdm2 and Mdm4 double heterozygous oocytes. These results demonstrated a critical prosurvival role for MDM2 in the oocytes. Moreover, they suggested a more tightened and rigorous regulatory mode for the MDM2/ MDM4-p53 network in female germ cells under stress situations. (Am J Pathol 2017, 187: 339-351; http://dx.doi.org/10.1016/j.ajpath.2016.09.023)
\end{abstract}

Folliculogenesis is a tightly regulated and continuous process, and it can be easily disrupted by inner or outside influences, which may lead to ovarian dysfunction, even infertility. Premature ovarian failure (POF), which affects approximately $1 \%$ of women, ${ }^{1}$ is defined as the occurrence of amenorrhea due to cessation of ovarian function in women younger than 40 years. Because many environmental factors have been linked to $\mathrm{POF},{ }^{2}$ understanding the mechanisms of stress responses in female germ cells, especially in the meiotically arrested oocytes in the primordial follicles, may help to improve our knowledge of this disease and to develop new strategies for better intervention.

The protein p53 is known for its roles as a tumor suppressor and a master regulator of cellular stress responses. It plays a crucial role in maintaining genomic stability and in suppressing tumor development in various organs. ${ }^{3}$ In somatic cells, p53 is maintained at low levels and is almost undetectable mainly through mouse double minute 2 (MDM2)-mediated ubiquitination and subsequent degradation. $^{4,5}$ MDM4, a homolog of MDM2, plays a nonoverlapping and synergistic role with MDM2 in inhibiting p53. ${ }^{6}$ In response to various stresses, including DNA damage, ribosome stress, hypoxia, and oncogenic activation, the associations between p53 and MDM2/ MDM4 become disrupted, leading to p53 stabilization and transcriptional activation, ${ }^{7}$ which resulted in cell cycle arrest, DNA repair, senescence, and apoptosis through expression of targeted genes such as p21, Puma, Noxa, and Bax. $^{8-11}$

Supported by National Basic Research Program of China grant 2010CB945102 (G.L.) and National Natural Science Foundation of China grants 30871265 and 31171305 (G.L.).

Disclosures: None declared. 
In females, p53 was found to regulate maternal reproduction by influencing blastocyst implantation through its target, leukemia inhibitory factor. ${ }^{12,13}$ However, how p53 regulates stress responses and female fertility in the germ cells is not completely understood. The ancient function of the p53 family might be the regulation of germ cell apoptosis. Studies in Caenorhabditis elegans indicated that p53 homolog CEP-1, which was most closely related to mammalian p53 family member p63, regulates germ cell apoptosis in response to DNA damage and meiotic recombination failure. ${ }^{14,15}$ In mice, p63 also appears to be required for apoptosis in oocytes of primordial follicles induced by irradiation or chemotherapeutic agents, such as cisplatin. ${ }^{16-19}$ Another study proposed that cisplatin was able to induce apoptosis of oocytes in the arrested primordial follicles by triggering a signaling network involving all three p53 family members (p53, p63, and p73), which was controlled by p63. ${ }^{20}$ More recently, it was shown that both the meiotically programed and exogenously induced DNA damage elicit a checkpoint requiring CHK2-mediated activation of both p63 and p53, with the deletion of $P 63$ alone insufficient for the longer term survival of the oocytes and rescue of infertility. ${ }^{21}$ Thus, these studies pointed to a combinatorial role of p63 and p53 in regulating apoptosis of the oocytes, quite distinct from the dominant role of p53 in somatic cells. The regulation of p53 functionality in the oocytes thus asks for further investigation.

In the present study, given the prominent role of MDM2 in regulating p53 activity in somatic cells, we aimed to examine the function of MDM2 in mouse oocytes at different stages of folliculogenesis and its impact on p53 signaling. We generated mutant mice with $M d m 2$-specific deletion in oocyte at different stages of folliculogenesis. These mice exhibited impeded fertility and early follicular loss phenotypes that were completely dependent on p53. We also found that MDM2 inhibitor Nutlin-3 was able to induce p53-dependent oocyte death. In addition, cancer therapeutic drugs 5-fluorouracil (5-FU) and doxorubicin were only able to activate p53 in oocytes in the presence of a combined reduction of gene dosages of $M d m 2$ and $M d m 4$. These results suggested the possibility of a more tightened control of the MDM2-p53 network in oocytes during folliculogenesis.

\section{Materials and Methods}

\section{Mice and Animal Care}

C57BL/6J-Gdf9-Cre and C57BL/6J-Zp3-Cre ${ }^{22,23}$ mice obtained from the Nanjing University-Nanjing Biomedical Institute (Nanjing, China) were crossed to $M d m 2^{F M}$, $M d m 4^{+/-}$mice, ${ }^{24,25}$ and $p 53^{\text {Flox }}\left(p 53^{F}\right)$ mice. ${ }^{26}$ All of the $M d m 2^{F M}, M d m 4^{+/-}$and $p 53^{F l o x}$ mice were backcrossed to C57BL/6 background for five generations. Mice were bred and maintained under specific pathogen-free conditions, and experiments were conducted in accordance with the Institutional Animal Care and Use Committee at the animal facility of Model Animal Research Center of Nanjing University, China.

\section{Fertility Studies of Female Mice}

To evaluate reproductive performance of the mice, six of each knockout and control group females were individually caged and bred to wild-type (WT) C57BL/6J males of known fertility at 5 or 6 weeks of age. The numbers of litters and pups produced were recorded over a 6-month period.

\section{Serum Hormone Analysis}

To evaluate serum hormone levels, mice were anesthetized by tribromoethanol, and blood was collected from the retroorbital of the mice. Blood of adult female Gdf9-Cre; $M d m 2^{F M / F M}$ and Zp3-Cre; $M d m 2^{F M / F M}$ mice at 2 to 4 months of age were collected randomly due to the lack of regular estrus cycles; blood of littermate control female mice were collected at the proestrus stage based on vaginal smears. ${ }^{27}$ Serum was prepared using blood collection tubes and stored at $-20^{\circ} \mathrm{C}$ until assayed. Follicle-stimulating hormone (FSH) levels were assayed by the FSH EnzymeLinked Immunosorbent Assay Kit (R\&D Systems, Minneapolis, MN) according to the manufacturer's protocols.

\section{Oocyte Collection}

To collect intact oocytes, oocytes from postnatal day (P) 7 to P14 mice were isolated by mechanical disruption of ovaries and follicles, followed by incubation in M2 medium (Sigma-Aldrich, St. Louis, MO) with trypsin $(1 \mathrm{mg} / \mathrm{mL}$; Sangon Biotech, Shanghai, China), collagenase $(1 \mathrm{mg} / \mathrm{mL}$; Sigma-Aldrich), hyaluronidase (1 mg/mL; Sigma-Aldrich), and DNaseI (10 $\mu \mathrm{g} / \mathrm{mL}$; Sigma-Aldrich) at $37^{\circ} \mathrm{C}$ for at least 10 minutes. Full-grown, germinal-vesicle-intact stage oocytes were collected by puncturing ovarian follicles from 3 week-old C57BL/6J female mice with a 30 -gauge needle in M2 medium. The surrounding granulosa cells were separated from the oocytes by pipetting. For each sample, at least 20 oocytes were collected for immunofluorescent staining or placed in $1 \mathrm{~mL}$ of TRIzol (Invitrogen, Carlsbad, CA), snap-frozen, and stored at $-20^{\circ} \mathrm{C}$ for RNA analysis.

\section{Gonadotropin-Induced Superovulation}

For superovulation experiments, four to five 3-week-old females (with the weight of 12 to $14 \mathrm{~g}$ ) were injected intraperitoneally with 5 IU of pregnant mare serum gonadotropin (Ningbo Sansheng Pharmaceutical Co., Ltd., Ningbo, China) and 46 hours later with 5 IU human chorionic gonadotropin (Ningbo Sansheng Pharmaceutical Co., Ltd.). Cumulus-oocyte complexes were isolated from the ampulla 16 hours after human chorionic 
gonadotropin injection and collected in M2 medium containing $1 \mathrm{mg} / \mathrm{mL}$ hyaluronidase (Sigma-Aldrich) to dissociate cumulus cells. The number of retrieved oocytes per female was recorded.

\section{RNA Extraction and qPCR Analysis}

RNA was isolated from the oocytes with TRIzol. For the RNA precipitation step, $20 \mu \mathrm{g}$ of glycogen (Invitrogen) was added into $0.5 \mathrm{~mL}$ isopropyl alcohol and incubated at $-20^{\circ} \mathrm{C}$ overnight. RNA was reverse transcribed into cDNA by using Moloney murine leukemia virus reverse transcriptase kit (Invitrogen) according to the manufacturer's instructions. The paired primers were as follows: Gapdh, 5'-TCCACCACCCTGTTGCTGTA-3' ${ }^{\prime}$ and $5^{\prime}$-TGAACGGGAAGCTCACTGG-3'; Puma, 5'-GCACCTAGTTGGGCTCCATTTCTG-3' and $5^{\prime}$-GTACGAGCG-GCGGAGACAAG- $3^{\prime} ; p 21$, $5^{\prime}$-AGGCAGCGTATATCAGGAG-3' and $5^{\prime}$-CCTGACAGATTTCTATCACTCCA-3'; Mdm2-exon5, 5'-ACGAGAAGCAGCAGCACATT- $3^{\prime}$ and $5^{\prime}$-ACTCGGGACTCCAAACACAT-3'; Bax, 5'-AGACAG-GGGCCTTTTTGCTAC$3^{\prime}$ and $5^{\prime}$-AATTCGCCGGAGACACTCG- $3^{\prime}$; Noxa, 5'-TCGCAAAAGAGCAGGATGAG- $3^{\prime}$ and $5^{\prime}$-CACTTTGTCTCCAATCCTCCG-3' (forward and reverse, respectively). The real-time quantitative PCRs (qPCRs) were performed with SYBR Green reagents (Invitrogen) on an ABI Prism Step-One Plus instrument (Applied Biosystems, Foster City, CA). Gapdh was used as endogenous control. The relative amount of target gene expression in knockout mice was calculated as fold change relative to littermate controls, after normalized to Gapdh.

\section{Quantification of Ovarian Follicles and Histologic Analysis}

Quantification of ovarian follicles was performed as previously described. ${ }^{28}$ Briefly, ovaries were fixed in $4 \%$ paraformaldehyde, dehydrated, and embedded in paraffin. To count the numbers of follicles, paraffin-embedded ovaries were serially sectioned at $6-\mu \mathrm{m}$ thickness and stained with hematoxylin and eosin for morphologic observation. Every fifth section was then analyzed by light microscopy for the presence of primordial, primary, secondary, and antral follicles. Only those follicles in which the nucleus of the oocyte was clearly visible were scored. For those values obtained by evaluating every fifth section, the cumulative follicle counts were multiplied by a factor of 5 to account for the fact that four-fifths of the ovary was not analyzed. Three to five ovaries were analyzed per genotype and per age.

\section{Immunofluorescence and Immunohistochemical Analyses}

Oocytes for immunofluorescence staining of p53 were collected and fixed in 2\% paraformaldehyde for 30 minutes at $4^{\circ} \mathrm{C}$. Then they were transferred to membrane permeabilization solution $[0.3 \%$ bovine serum albumin, $0.1 \%$ Triton X-100 in phosphate-buffered saline (PBS)] for 10 minutes. After permeabilization, oocytes were washed three times in $1 \times$ PBS with $0.1 \%$ Tween-20, 5 minutes each. Then the oocytes were placed in blocking solution ( $0.3 \%$ bovine serum albumin, $0.1 \%$ Tween-20, PBS) for 2.5 hours. Oocytes were incubated with primary antibodies against p53 (dilution 1:500; CM5; Novocastra, Newcastle, United Kingdom) overnight at $4^{\circ} \mathrm{C}$. Then oocytes were washed and followed by secondary antibodies conjugated with cyanine 3/fluorescein isothiocyanate (Jackson ImmunoResearch Laboratories, West Grove, PA) for 2 hours at room temperature; omission of the primary antibody served as control in which case no signal was observed. The DNA was stained with DAPI (1 mg/mL; D6584; Sangon Biotech). The oocytes were then washed and mounted. For immunofluorescent analysis of p53 with frozen sections, mouse ovaries were washed with cold PBS and fixed in $4 \%$ paraformaldehyde for 1 hour at $4^{\circ} \mathrm{C}$. The samples were washed three times in $1 \times$ PBS, 10 minutes each at $4^{\circ} \mathrm{C}$. Then ovaries were washed in $30 \%$ sucrose $(1 \times \mathrm{PBS})$ at $4^{\circ} \mathrm{C}$ until they sunk to the bottom of the vial. The processed ovaries were then embedded in OCT and sectioned $(10 \mu \mathrm{m}$ in thickness). For immunofluorescent staining, frozen sections were incubated at $4{ }^{\circ} \mathrm{C}$ overnight with antibodies for $\mathrm{p} 53$ (dilution 1:500; CM5; Novocastra). Fluorescence was evaluated using a Leica (Wetzlar, Germany) TCS SP2 confocal laser scanning microscope or Olympus (Tokyo, Japan) FV 1000 confocal microscope.

For immunofluorescence/immunohistochemical analysis on paraffin sections, antigen retrieval was processed by incubating the slides in $0.01 \mathrm{~mol} / \mathrm{L}$ sodium citrate buffer $(\mathrm{pH}$ 6.0) or Tris-EDTA buffer ( $\mathrm{pH} 9.0,0.05 \%$ Tween 20) for 25 minutes in a steamer. Primary antibodies were as follows: anti-p53 (dilution 1:500; CM5; Novocastra), anti-cleaved caspase-3 (dilution 1:200; Asp 175; Cell Signaling Technology, Danvers, MA), anti-DEAD (Asp-Glu-Ala-Asp) box polypeptide 4/MVH (dilution 1:200; ab13840; Abcam, Cambridge, MA), anti-proliferating cell nuclear antigen (PCNA) (dilution 1:200; PC10; Cell Signaling Technology).

\section{Western Blot Analysis}

Mouse ovaries were lysed in RIPA buffer supplemented with protease and phosphatase inhibitor cocktail tablets (Roche, Basel, Switzerland). The membranes were incubated with anti-PCNA (dilution 1:1000; PC10; Cell Signaling Technology), and appropriate secondary antibody (dilution 1:3000; Sigma-Aldrich) sequentially.

\section{In Vitro Ovarian Culture}

Ovaries were removed from P5 female mice and placed on a piece of Millicell-CM membrane inserts $(0.4 \mu \mathrm{m}$; Millipore, Bedford, MA) covered with only a thin film of culture medium. The medium for organ culture was Dulbecco's 
modified Eagle's medium/F12 (HyClone, Logan, UT) supplemented with $1 \mathrm{mg} / \mathrm{mL}$ bovine serum albumin (SigmaAldrich), $1 \mathrm{mg} / \mathrm{mL}$ Albumax (Gibco BRL, Grand Island, $\mathrm{NY}$ ), $50 \mu \mathrm{g} / \mathrm{mL}$ ascorbic acid (Sigma-Aldrich), $5 \mathrm{U} / \mathrm{mL}$ penicillin $/ 5 \mu \mathrm{g} / \mathrm{mL}$ streptomycin (HyClone), and $27.5 \mathrm{mg} / \mathrm{mL}$ transferrin (Sigma-Aldrich). The organ cultures were maintained at $37^{\circ} \mathrm{C}$ and $5 \% \mathrm{CO}_{2}$ in modular incubation chambers (Thermo Scientific, Rockford, IL). For those cultures lasting $>2$ days, the medium was removed and fresh medium was added every 2 days. Three to five ovaries per treatment were cultured for each experiment. Experiments were repeated 3 times.

\section{Statistical Analysis}

Data were expressed as means \pm SEM. For comparisons between two groups, statistical significance was determined using unpaired two-tailed $t$-test. $P<0.05$ was considered statistically significant, and $P<0.01$ and $P<0.001$ were statistically highly significant.

\section{Results}

Deletion of Mdm2 in 0ocytes of the Primordial Follicles Results in Infertility in Female Mice

To determine the function of MDM2 in oocyte survival and growth, we crossed $M d m 2^{F M / F M}$ mice, in which the exon 5 and 6 of the $M d m 2$ gene were flanked by loxP sites ${ }^{24}$ with Gdf9-Cre transgenic mice. Expression of Gdf9-Cre is first detected in the oocytes of primordial follicles at P3. ${ }^{22}$ Oocytes from $\mathrm{Gdf9}$-Cre; $M d m 2^{F M / F M}$ females displayed $>80 \%$ decrease in $M d m 2$ mRNA levels compared with $M d m 2^{F M / F M}$ controls at P7 (Supplemental Figure S1A). To investigate whether the deletion of $M d m 2$ in oocytes influenced the reproductive performance of the mice, females were bred to WT males beginning at 5 weeks of age and continuously for 6 months. There were a steady average number of pups per litter (Figure 1A) and increased cumulative number of offspring produced by the $M d m 2^{F M / F M}$ females (Figure 1B). In contrast, only two of six $G d f 9-C r e ; M d m 2^{F M / F M}$ females were able to give birth to a small number of offspring in the first month of mating, with all of the six mice producing no offspring thereafter (Figure 1, A and B). Consistently, we also found drastically reduced ovarian size (Figure 1C) and weight (Figure 1D) in Gdf9-Cre; $M d m 2^{F M / F M}$ females at the age of 6 weeks, when the female mice normally reach their sexual maturity. Ovaries of $M d m 2^{F M / F M}$ mice contained both growing follicles and numerous primordial follicles that would preserve their fertility for the rest of the reproductive life. In comparison, there was a complete lack of follicular structures in the ovaries of $G d f 9-C r e ; M d m 2^{F M / F M}$ mice with only corpus luteum remaining (Figure $1 \mathrm{C}$ ). Moreover, elevated level of FSH, which was used for diagnosis of POF in humans, ${ }^{29}$ was found in sera of 2- to 3-month-old
Gdf9-Cre; $M d m 2^{F M / F M}$ mice (Figure 1E). Collectively, these results showed that deletion of $M d m 2$ in oocytes of primordial follicles led to a phenotype resembling human $\mathrm{POF}$ in mice.

\section{Rapid Depletion of Primordial Follicles in Gdff-Cre; $M d m 2^{F M / F M}$ Mice}

The follicular resource available in the ovary is a direct determinant of female reproductive life span. ${ }^{30,31}$ To investigate how the absence of $M d m 2$ in oocytes gradually led to infertility, ovarian structures of Gdf9-Cre; $M d m 2^{F M / F M}$ and $M d m 2^{F M / F M}$ females at P5, P8, and P25 were examined. Ovaries of both $G d f 9-C r e ; M d m 2^{F M / F M}$ and $M d m 2^{F M / F M}$ females at P5 contained similar numbers of follicles, including mostly primordial follicles with small oocytes surrounded by flattened pregranulosa cells and a few activated follicles with enlarged oocytes surrounded by one or more layers of cubic granulosa cells (Figure 2, A and B). However, at P8, Gdf9-Cre; $M d m 2^{F M / F M}$ ovaries contained few primordial follicles and a significantly reduced number of activated follicles, including primary and secondary follicles (Figure 2, C and D). At P25, the Gdf9-Cre; $M d m 2^{F M / F M}$ ovaries appeared much smaller with the markedly reduced numbers of follicles. Healthy-looking primordial follicles and primary and secondary follicles in Gdf9-Cre; $M d m 2^{F M / F M}$ ovaries were scarce, corresponding to $1.9 \%, 0.4 \%, 2.2 \%$ of those in $M d m 2^{F M / F M}$ ovaries, respectively (Figure 2, E and F). Consistently, the weight of Gdf9-Cre; $M d m 2^{F M / F M}$ ovaries was significantly reduced compared with the controls at both P25 and 3 months of the age (Figure 2G).

To study the rapid depletion of primordial follicles, immunohistochemical analysis of cleaved caspase- 3 was performed to evaluate apoptosis. Apoptosis, particularly in oocytes of primordial follicles, was significantly elevated in ovaries of Gdf9-Cre; $M d m 2^{F M / F M}$ mice (Figure 2, $\mathrm{H}$ and I).

\section{Deletion of Mdm2 in 0ocytes of the Primary Follicles} Leads to Follicular Loss and Decreased Fertility in Mice

The rapid depletion of primordial follicles in Gdf9-Cre; $M d m 2^{F M / F M}$ ovaries makes it difficult to address the function of MDM2 in oocytes at other stages of folliculogenesis. To circumvent this problem, we generated mice with specific $M d m 2$ deletion in oocytes of growing follicles using $\mathrm{Zp3}$-Cre allele. The Zp3-Cre activity, which is first detected in P5 mouse ovaries, is restricted in oocytes of primary and further developed follicles. ${ }^{32}$ qPCR analysis showed that oocytes from Zp3-Cre; $M d m 2^{F M / F M}$ females displayed a $48 \%$ and $89 \%$ decrease in Mdm2 mRNA levels at P7 and P12, respectively (Supplemental Figure S1B).

Fertility testing was performed on $\mathrm{Zp} 3-\mathrm{Cre} ; \mathrm{Mdm} 2^{\text {FM/FM }}$ and $M d m 2^{F M / F M}$ control female mice. Compared with control females, Zp3-Cre; Mdm2 $2^{F M / F M}$ females gave birth 
A

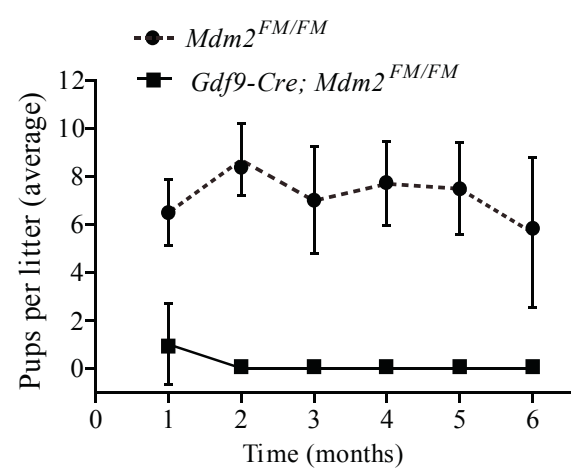

B

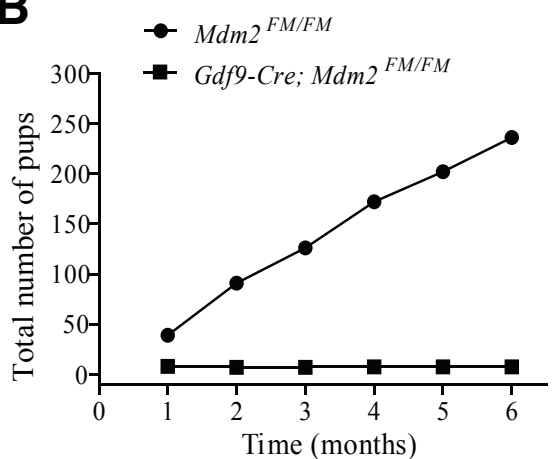

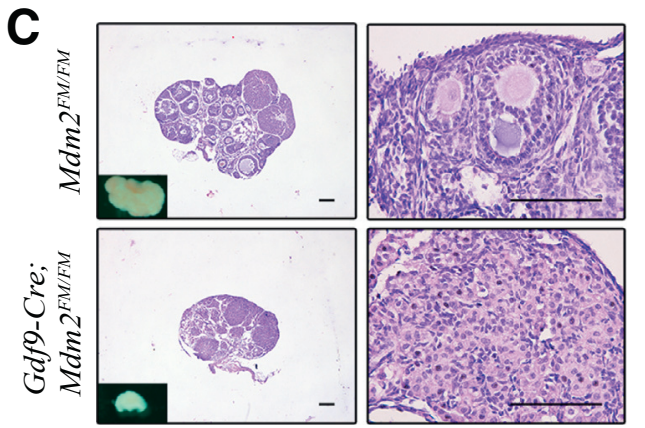
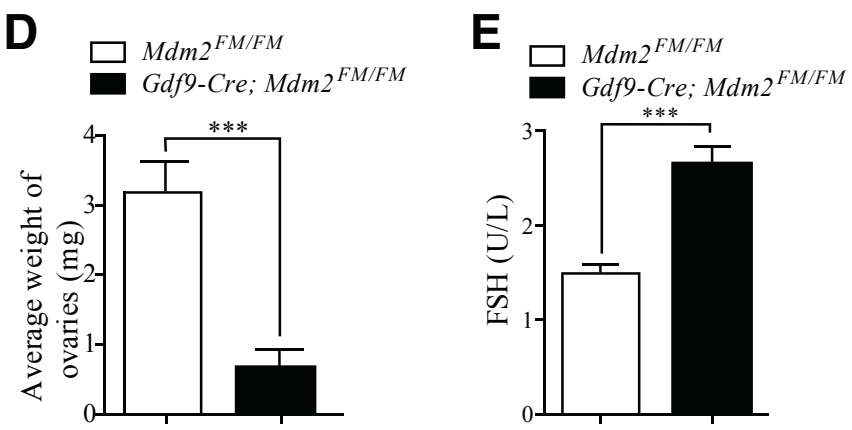

Figure $1 M d m 2$ deletion mediated by Gdf9-Cre resulted in infertility in female mice. A and B: Six Gdf9-Cre; Mdm2 $2^{F M / F M}$ and $M d m 2^{F M / F M}$ female mice were individually caged and bred to WT C57BL/6J males of known fertility over a 6 -month period. Average number of pups per litter from the mating in each month (A) and the cumulative number of pups over a 6-month period (B) are shown. C: H\&E-stained ovary sections from 6-week-old Gdf9-Cre; Mdm2 $2^{F M / F M}$ and $M d m 2^{F M / F M}$ mice. Inset: images of ovaries by a light microscope. D: Average weight of ovaries obtained from 6-week-old Gdf9-Cre; $M d m 2^{F M / F M}$ and $M d m 2^{F M / F M}$ mice. E: FSH levels in sera of young adult (2- to 3-month-old) Gdfg-Cre; Mdm2 $2^{F M / F M}$ and Mdm2 $2^{F M / F M}$ mice. Data are expressed as means \pm SEM. $n=6$ (A, B, and D); $n=9$ (E). ${ }^{* * *} P<0.001$. Scale bar $=200 \mu \mathrm{m}$ (C). FSH, follicle-stimulating hormone; H\&E, hematoxylin and eosin; WT, wild-type.

to a significantly reduced number of pups per litter and total number of pups in the 6-month period of breeding (Figure 3, A and B). Zp3-Cre; Mdm2 $2^{F M / F M}$ females also showed a significant reduction in ovarian weight compared with control mice at both P25 and 3 months of age (Figure 3C), although the phenotype was less severe than the Gdf9-Cre; Mdm2 $2^{F M / F M}$ mice (Figure 2G). Follicular development of Zp3-Cre; $M d m 2^{F M / F M}$ and control mice was further examined. The number of primordial follicles was slightly higher in $\mathrm{Zp3}$-Cre; $\mathrm{Mdm} 2^{F M / F M}$ mouse ovaries at P8, P25, and 3 months of age, although the difference was not statistically significant (Figure 3, D-I). At P8, the numbers of primary and secondary follicles were only slightly decreased (Figure 3, D and E). At P25, except for primordial follicles, the numbers of other follicles were all decreased in $\mathrm{Zp} 3-\mathrm{Cre}$; $\mathrm{Mdm} 2^{F M / F M}$ mice, with the primary follicles showing a milder reduction than the secondary follicles, corresponding to $41 \%$ and $4.7 \%$ of those in control ovaries, respectively (Figure 3, F and G). In addition, Zp3Cre; $M d m 2^{F M / F M}$ ovaries contained many degenerating oocytes and atretic follicles (Figure 3F). At 3 months of age, plenty of primary follicles were still found in the outer region of the medulla of the $\mathrm{Zp3}-\mathrm{Cre}$; $\mathrm{Mdm} 2^{F M / F M}$ ovary, yet only few secondary and antral follicles remained (Figure 3, H and I). Collectively, these results suggested the possibility that either the progression of the primary follicles to the secondary follicles was inhibited in the Zp3-Cre; $M d m 2^{F M / F M}$ mice or that the oocytes of primary follicles were less sensitive to $M d m 2$ deletion than those of the secondary follicles. In either case, the follicular development and survival beyond the stage of primary follicles were severely affected in Zp3-Cre; $M d m 2^{F M / F M}$ mice. Consistent with their ovarian dysfunction, Zp3-Cre; $M d m 2^{F M / F M}$ mice also displayed a significant increase in the serum FSH levels (Figure 3J).

It is well known that the status of oocyte closely influences the growth and survival of their surrounding granulosa cells. ${ }^{33,34}$ There was reduced proliferation of granulosa cells in the growing follicles of $\mathrm{Zp3}-\mathrm{Cre}$; $M d m 2^{F M / F M}$ ovaries, as shown by immunohistochemical staining for PCNA in the ovarian sections (Supplemental Figure S2A). Western blot analysis also confirmed a dramatic decrease of PCNA in the Zp3-Cre; $M d m 2^{F M / F M}$ ovaries (Supplemental Figure S2B). Meanwhile, apoptosis of granulosa cells, which was closely associated with follicular atresia, ${ }^{35}$ was also found increased in the P25 Zp3-Cre; Mdm2 $2^{F M / F M}$ ovaries (Supplemental Figure S2C). These results collectively suggested a suppression of follicular growth and an increase in follicular atresia in the Zp3-Cre; $M d m 2^{F M / F M}$ mice. 

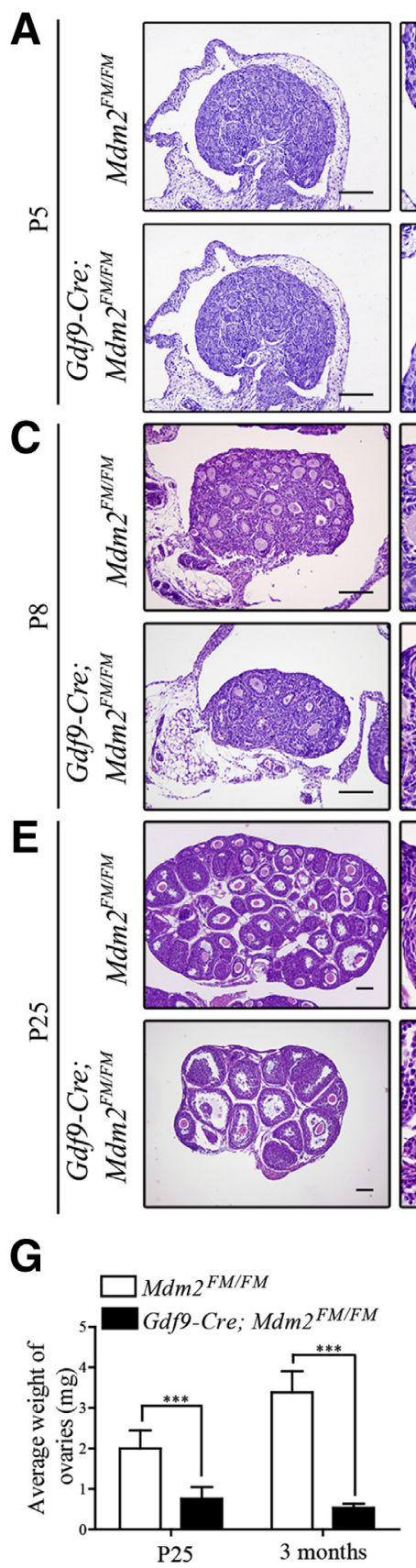
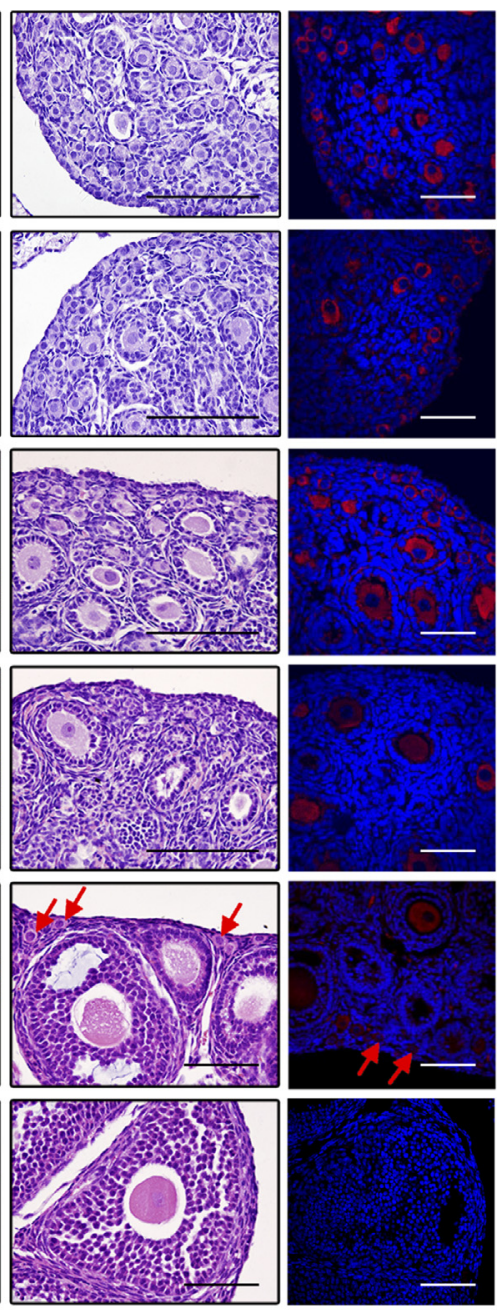

H
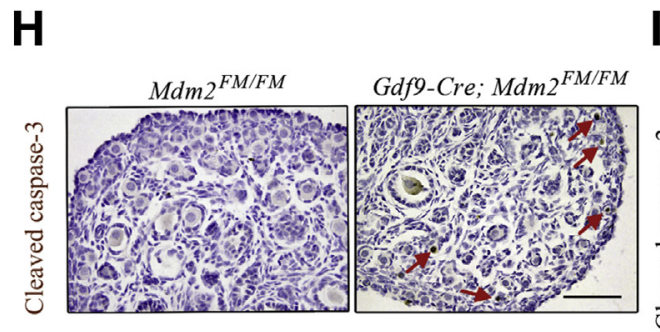

B

$\mathbf{F}$
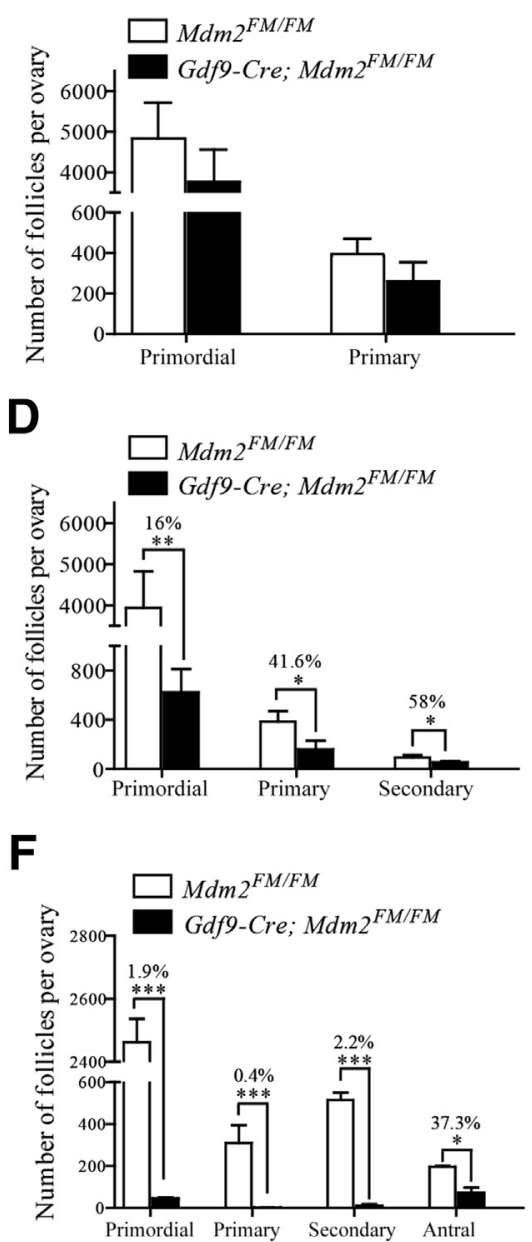

| $\square M d m 2^{F M / F M}$

Gdf9-Cre; Mdm2 $2^{F M / F M}$

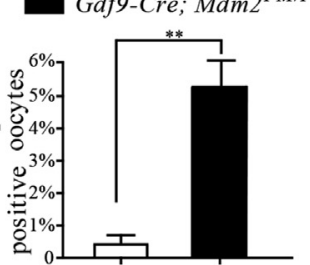

Figure 2 Deletion of Mdm2 in oocytes led to a rapid depletion of primordial follicles in the Gdfg-Cre; Mdm2 $2^{F M / F M}$ mice. A, C, and E: Morphologic analysis of ovaries from Gdfg-Cre; $M d m 2^{F M / F M}$ and $M d m 2^{F M / F M}$ littermates. Ovaries from P5 (A), P8 (C), and P25 (E) Gdf9-Cre; Mdm2 $2^{F M / F M}$ and $M d m 2^{F M / F M}$ mice were embedded in paraffin, and serial sections of $6-\mu \mathrm{m}$ thickness were prepared and stained with H\&E. Follicles were clearly identified by immunofluorescence staining for oocyte marker DDX4 (right panel, red). Arrows in E depict primordial follicles. B, D, and F: The numbers of follicles per ovary in P5 (B), P8 (D), and P25 (F) Gdfg-Cre; Mdm2 $2^{F M / F M}$ and $M d m 2^{F M / F M}$ mice were quantified as described in Materials and Methods. G: Average weight of ovaries obtained from P25 and 3-month-old Gdf9-Cre; $M d m 2^{F M / F M}$ and $M d m 2^{F M / F M}$ mice. H: Representative micrographs of immunohistochemical staining of cleaved caspase3 from ovaries of P5 Gdf9-Cre; $M d m 2^{F M / F M}$ and $M d m 2^{F M / F M}$ mice. Arrows in $\mathbf{H}$ depict apoptotic oocytes. I: Quantification of cleaved caspase-3-positive oocytes. Data are expressed as means \pm SEM. $n=3$ (B, D, and I); $n=4(\mathbf{F}) ; n=6(\mathbf{G})$. ${ }^{*} P<0.05,{ }^{*} P<0.01$, and ${ }^{* * *} P<0.001$. Scale bars: $100 \mu \mathrm{m}$ $(\mathbf{A}, \mathbf{C}$, and E); $20 \mu \mathrm{m}(\mathbf{H})$. DDX4, DEAD (Asp-Glu-Ala-Asp) box polypeptide 4; H\&E, hematoxylin and eosin; $\mathrm{P}$, postnatal day.

p53 Activation Is Responsible for the Follicular Loss and Impeded Fertility Caused by the Deletion of Mdm2 in Mice

MDM2 is the main negative regulator of p53 in somatic cells. To investigate whether loss of $M d m 2$ resulted in p53 activation in female germ cells, immunofluorescence analyses were performed with both the ovarian sections (Figure 4A) and isolated oocytes (Figure 4, C and E). Although p53 expression was undetectable in the oocytes of the control group, $M d m 2$ loss resulted in significant p53 nuclear accumulation in oocytes from primordial and 


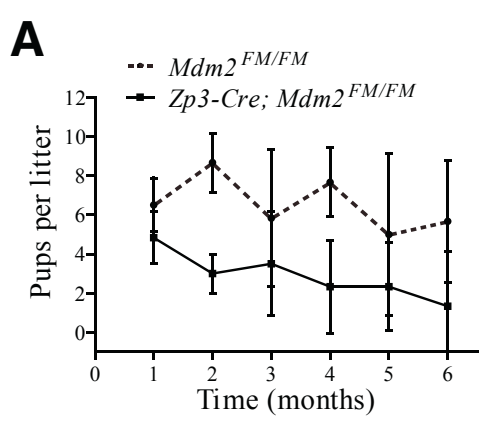

D

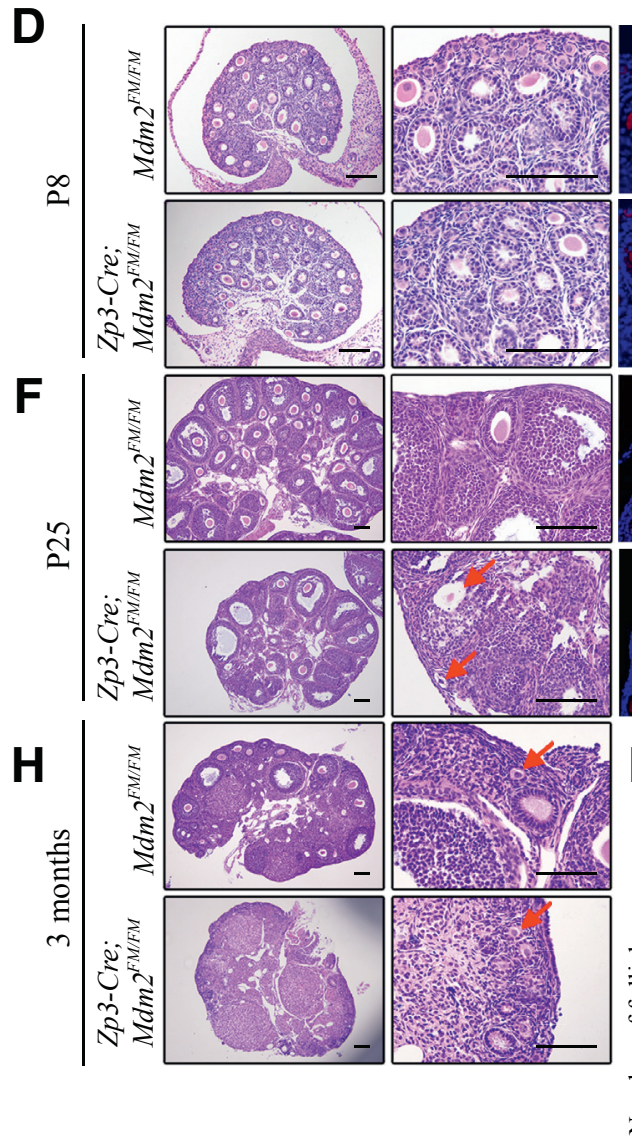

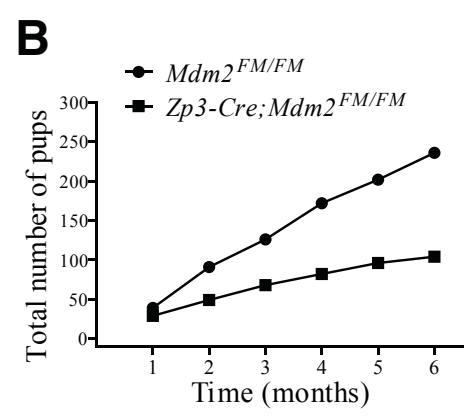

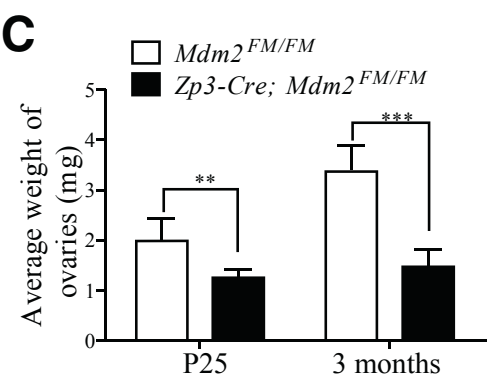

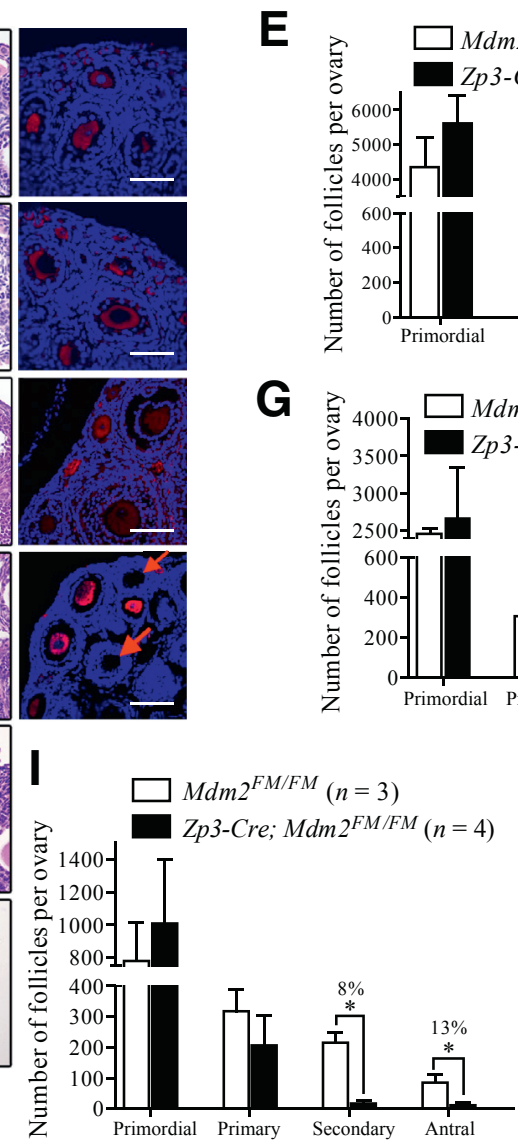
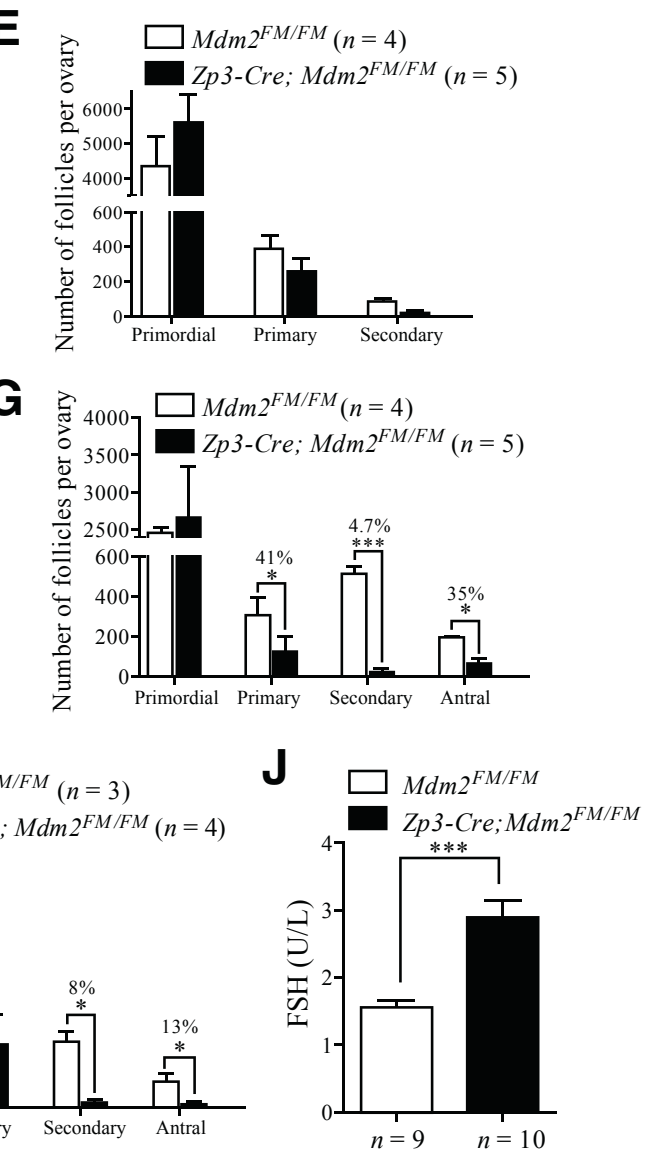

Figure 3 Deletion of $M d m 2$ in oocytes of the primary follicles led to follicular loss and decreased fertility in the $Z p 3-C r e ; M d m 2^{F M / F M}$ mice. A and B: $Z p 3-C r e$; $M d m 2^{F M / F M}$ and $M d m 2^{F M / F M}$ female mice were individually caged and bred to WT C57BL/6J males of known fertility over a 6 -month period. Average number of pups per litter from the matings in each month (A) and the cumulative number of pups over a 6-month period (B) are shown. C: Average weight of ovaries obtained from P25 and 3-month-old Zp3-Cre; Mdm2 $2^{F M / F M}$ and Mdm2 $2^{F M / F M}$ mice. D, F, and H: Morphologic analysis of ovaries from Zp3-Cre; Mdm2 $2^{F M / F M}$ and $M d m 2^{F M / F M}$ mice at P8 (D), P25 (F), and 3 months of age (H). Follicles are clearly identified by immunofluorescent staining for oocyte marker DDX4 (right panel, red). Arrows in $\mathbf{F}$ depict oocytes undergoing apoptosis or degeneration. Arrows in $\mathbf{H}$ depict primary follicles. E, G, and I: Quantification of follicle numbers in ovaries of P8 (E), P25 (G), and 3-month-old (I) Zp3-Cre; Mdm2 $2^{F M / F M}$ and Mdm2 ${ }^{F M / F M}$ mice as described in Materials and Methods. J: FSH levels in sera of 3- to 4-month-old Zp3-Cre; Mdm2 $2^{F M / F M}$ and $M d m 2^{F M / F M}$ mice. Data are expressed as means \pm SEM. $n=6$ unless specified otherwise. ${ }^{*} P<0.05,{ }^{*} P<0.01$, and ${ }^{* * * P}<0.001$. Scale bar $=200 \mu \mathrm{m}$. FSH, follicle-stimulating hormone; $P$, postnatal day; WT, wild-type.

growing follicles of the Gdf9-Cre; $M d m 2^{F M / F M}$ mice (Figure 4, A-D). An increase in the nuclear intensity of p53 was also found in most oocytes isolated from the Zp3-Cre; $M d m 2^{F M / F M}$ mice (Figure 4, E and F).

Consistent with the p53 activation, qPCR analysis showed increased expression of proapoptotic genes Puma, Noxa, Bax, and the cell cycle inhibitory gene $p 21$, all of which are p53 transcriptional target genes, in ovaries of both
Gdf9-Cre; $M d m 2^{F M / F M}$ and Zp3-Cre; $M d m 2^{F M / F M}$ mice (Figure 4, G and $\mathrm{H}$ ).

To explore whether p53 activation in oocytes is responsible for the ovarian phenotypes displayed in the Gdf9-Cre; $M d m 2^{F M / F M}$ and $Z p 3-C r e ; M d m 2^{F M / F M}$ mice, we generated oocyte-specific $M d m 2$ and p53 double knockout mice. Fertility testing showed that the drastically reduced fertility caused by the $M d m 2$ deletion was completely rescued in 
A
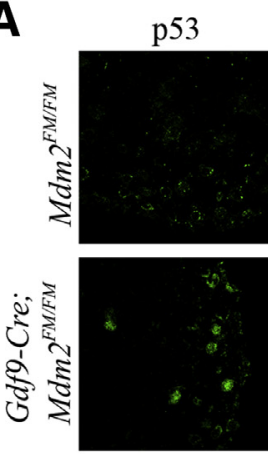

C
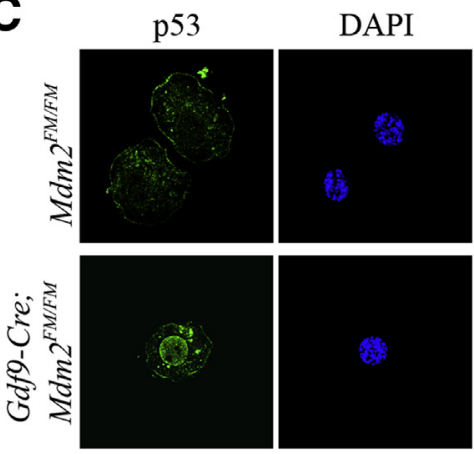

$\mathbf{E}$
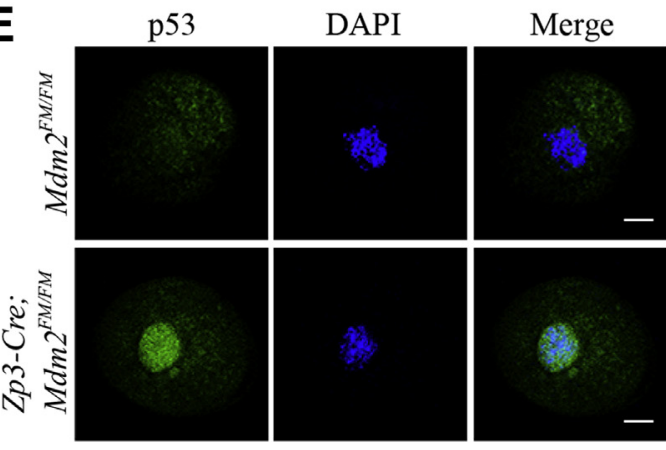

G

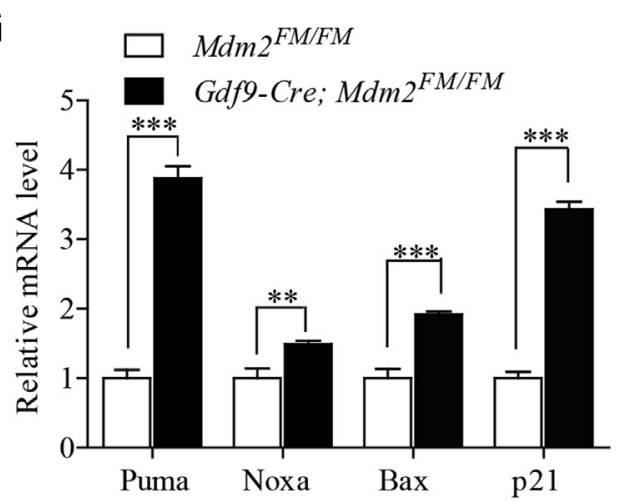

Merge

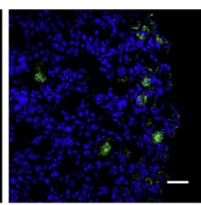

Merge

Merge
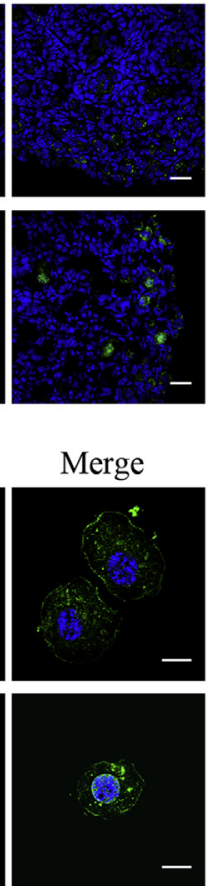

F

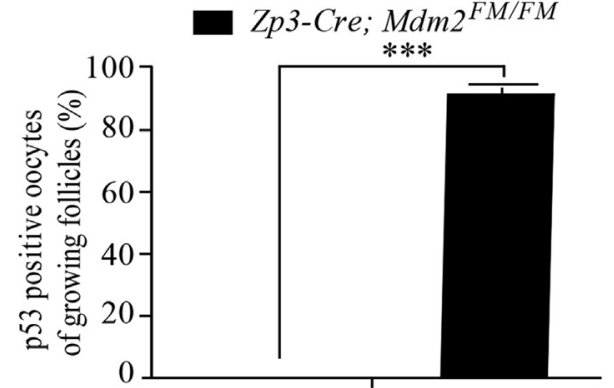

H

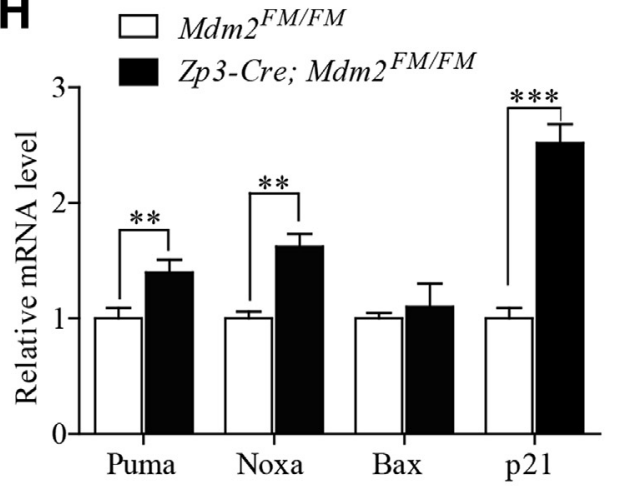

Figure 4 Up-regulated p53 activity in oocytes of Gdf9-Cre; Mdm2 $2^{F M / F M}$ and Zp3-Cre; Mdm2 $2^{F M / F M}$ female mice. A: Representative images of p53 immunofluorescence (green) in frozen sections of ovaries from P7 Gdf9-Cre; Mdm2 $2^{F M / F M}$ and $M d m 2^{F M / F M}$ mice. B: Quantification of p53-positive-stained oocytes in the ovarian sections. C: Representative images of p53 immunofluorescence (green) in oocytes isolated from P7 Gdf9-Cre; Mdm2 $2^{F M / F M}$ and $M d m 2^{F M / F M}$ mice. D: Quantification of p53-positive-stained oocytes. E: Representative images of p53 immunofluorescence (green) in isolated oocytes from P12 Zp3-Cre $M d m 2^{F M / F M}$ and $M d m 2^{F M / F M}$ mice. F: Quantification of p53-positive-stained oocytes. G: qPCR analysis of mRNA expression in oocytes isolated from P7 Gdf9-Cre; $M d m 2^{F M / F M}$ and $M d m 2^{F M / F M}$ mice. Approximately 60 oocytes were collected in each group. H: qPCR analysis of mRNA expression in oocytes isolated from P12 $Z p 3-C r e ; M d m 2^{F M / F M}$ and Mdm2 $2^{F M / F M}$ mice. Approximately 40 oocytes were collected in each group. Data are expressed as means \pm SEM. $n=3$. ${ }^{* * P}<0.01$, ${ }^{* * *} P<0.001$. Scale bars: $25 \mu \mathrm{m}(\mathbf{A}) ; 10 \mu \mathrm{m}(\mathbf{C}$ and $\mathbf{E})$. P, postnatal day; qPCR, real-time quantitative PCR. 
both Gdf9-Cre; $M d m 2^{F M / F M} ; \quad p 53^{F / F}$ and Zp3-Cre; $M d m 2^{F M / F M} ; p 53^{F / F}$ mice (Supplemental Figure S3, A and B). There were no differences in either ovarian size or structure of these two groups of mice compared with the $M d m 2^{F M / F M} ; \quad p 53^{F / F}$ control group at both $\mathrm{P} 25$ (Supplemental Figure S3, C and D) and even 8 months of age (Supplemental Figure S3, E and F), indicating normal follicular development in these mice. p53 was undetectable in the Gdf9-Cre; $M d m 2^{F M / F M} ; p 53^{F / F}$ and Zp3-Cre; $M d m 2^{F M / F M} ; p 53^{F / F}$ oocytes, further validating the specificity of p53 nuclear staining found in the Gdf9-Cre; $M d m 2^{F M / F M}$ and Zp3-Cre; $\quad M d m 2^{F M / F M}$ oocytes (Supplemental Figure S3G). Meanwhile, expression levels of Puma, Noxa, Bax, and $p 21$ in the Gdf9-Cre; Mdm $2^{F M / F M}$; $p 53^{F / F}$ and $\mathrm{Zp} 3$-Cre; $M d m 2^{F M / F M} ; p 53^{F / F}$ oocytes were also comparable with those in control littermates (Supplemental Figure S3, H and I). Collectively, these results demonstrated that the defects in the follicular development and survival observed in the Gdf9-Cre; $M d m 2^{F M / F M}$ and Zp3-Cre; $M d m 2^{F M / F M}$ mice were completely dependent on $\mathrm{p} 53$, indicating that regulation of $\mathrm{p} 53$ by MDM2 is indispensable during mouse folliculogenesis.

\section{p53-Dependent 0ocyte Death Induced by Nutlin-3}

Nutlin-3 is a small molecule chemical compound that binds tightly and selectively to the p53-interaction domain of MDM2. ${ }^{36}$ Treatment with Nutlin-3 has been shown to restore p53 activation for subsequent induction of apoptosis, senescence, or reversible cell cycle arrest in various model systems. ${ }^{37,38}$ However, the potential side effect of Nutlin-3 on female reproduction is still unclear. To determine whether Nutlin-3 could induce oocyte death similarly as genetic deletion of $M d m 2$, WT mouse ovaries at P5 were cultured with various doses of Nutlin-3 (2 to $10 \mu \mathrm{mol} / \mathrm{L}$ ) for 4 days. With 2 and $5 \mu \mathrm{mol} / \mathrm{L}$ of Nutlin-3 treatment, most of the primordial follicles were intact, and a few unhealthy follicles could be distinguished by pyknosis of granulosa cells and intense eosinophilic staining of oocytes (Figure 5A). Treatment with $10 \mu \mathrm{mol} / \mathrm{L}$ of Nutlin-3, however, eliminated most of the follicles with only rare healthy oocytes remaining (Figure 5A). Forty-eight hours after treatment with $10 \mu \mathrm{mol} / \mathrm{L}$ Nutlin-3, there was a significant increase in cleaved caspase-3-positive oocytes (Figure 5B). p53 was also found to be accumulated in the nucleus of the oocyte in the Nutlin-3-treated ovaries (Figure 5C).

To validate the function of p53 in oocyte in Nutlin3-induced follicular loss, ovaries with oocyte-specific deletion of $p 53$ were also cultured in the presence of 10 $\mu \mathrm{mol} / \mathrm{L}$ Nutlin-3. Four days after the treatment, the prominent loss of normal primordial and primary follicles was effectively suppressed by the $p 53$ deletion (Figure 5, D and E). Although only $6.8 \%$ of primordial follicles remained after 4 days of $10 \mu \mathrm{mol} / \mathrm{L}$ Nutlin-3 treatment in the control group, approximately $67 \%$ of primordial follicles with oocyte-specific p53 deletion survived. Nutlin-3 treatment also eliminated $93 \%$ of primary follicles in the control mouse ovaries. In contrast, the number of primary follicles was not significantly different in the untreated and treated Gdf9-Cre; $p 53^{F / F}$ ovaries (Figure 5E). These results suggested that drugs that directly disrupted the p53-MDM2 interaction were able to activate p53 in the oocytes and to induce follicular loss in mice.

\section{5-FU and Doxorubicin Are Able to Activate p53 in Mdm2 and Mdm4 Double Heterozygous Oocytes}

Many chemotherapeutic drugs, such as 5-FU and doxorubicin, have the ability to attenuate the MDM2-p53 interaction to activate p53 in somatic cells through various signaling mechanisms. To investigate whether these drugs could activate p53 in oocytes in the primordial and growing follicles, WT and control mice at P10 were treated with 5-FU and doxorubicin. Twenty-four hours after a single i.p. injection of either $5-\mathrm{FU}(15 \mathrm{mg} / \mathrm{kg})$ or doxorubicin $(20 \mathrm{mg} / \mathrm{kg})$, there was a significant increase in p53-positive immunostaining in the granulosa cells of the follicles (Figure 6, A and B). However, no p53-positive staining was detected in the oocytes of these mice (Figure 6, A and B), suggesting that these drugs were insufficient in mounting a robust $\mathrm{p} 53$ response in the short term.

Previous studies reported that MDM2 and MDM4 play synergistic roles in p53 inhibition. ${ }^{39,40}$ To investigate whether p53 could be activated by chemotherapeutic drugs in the presence of reduced gene dosages of $M d m 2$ and $M d m 4$, mice double heterozygous for $M d m 2$ and $M d m 4$ in oocytes (Gdf9-Cre; $\mathrm{Mdm} 2^{\mathrm{FM} /+} ; \mathrm{Mdm} 4^{+/-}$, referred as Gdf9-Cre; $M M$ ) were derived by crossing Gdf9-Cre mice with the $M d m 2^{F M / F M} ; M d m 4^{+/-}$mice and analyzed for $\mathrm{p} 53$ activation and sensitivity to drug treatment. Both the p53 immunostaining (Supplemental Figure S4A) and expression of p53 target genes (Supplemental Figure S4B) in the oocytes were not altered in the $G d f 9-C r e ; M M$ mice without the drug treatments. In addition, the Gdf9-Cre; $M M$ mice displayed normal superovulation rates at 3 weeks of age (Supplemental Figure S4C). Analysis on ovarian structure also showed normal follicular development in these mice (Supplemental Figure S4D). However, 24 hours after a single i.p. injection of either 5-FU or doxorubicin, there was increased p53 accumulation in both granulosa cells and nucleus of oocytes in the P10 Gdf9-Cre; MM mice but not in oocytes of the control littermates, including Gdf9-Cre; $M d m 2^{F M /+}, M d m 2^{F M /+} ; M d m 4^{+/-}$, and $M d m 2^{F M /+}$ mice (Figure 6, A and B). Moreover, accelerated oocyte death or degeneration was found in the 5-FU-treated Gdf9-Cre; MM mice, whereas most of the oocytes remained healthy in the control mice with the same treatment (Figure 6C). Cleaved caspase-3 staining also confirmed that oocytes from the small follicles of $\mathrm{Gdf9-Cre}$; $M M$ mice were prone to apoptosis by the 5-FU treatment (Supplemental Figure S5). These results suggested that a combined and dosedependent inhibition of p53 by MDM2 and MDM4 may 
A

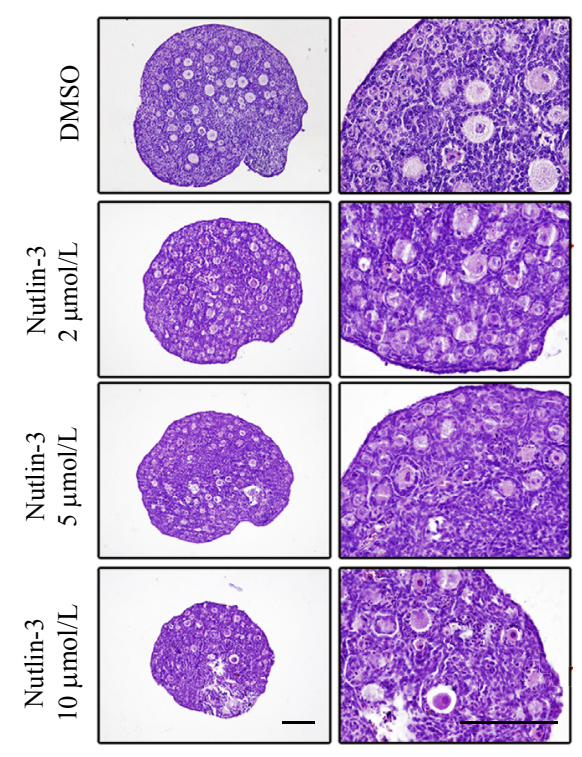

B

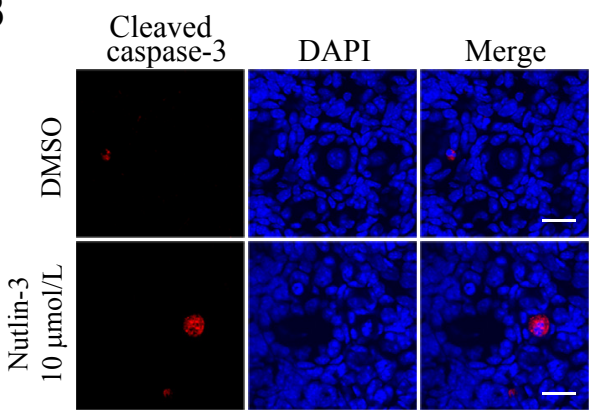

C

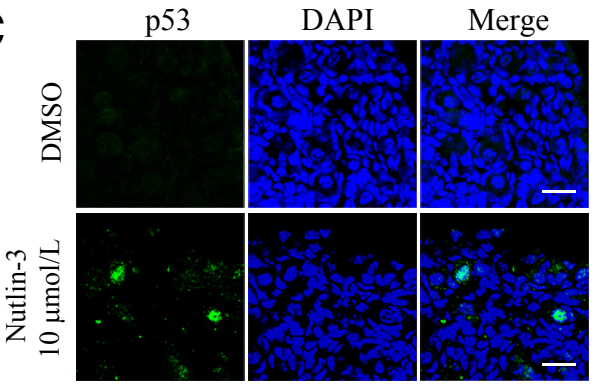

D

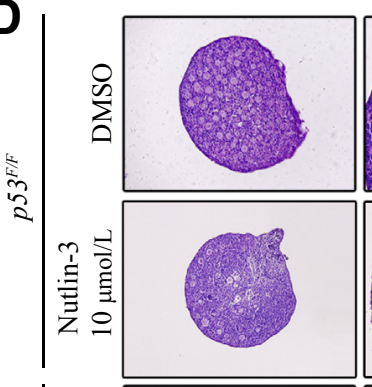

|̇̃

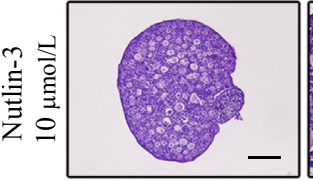

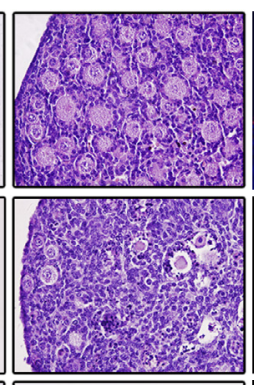

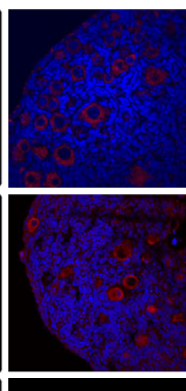

\section{$\mathbf{E}$}
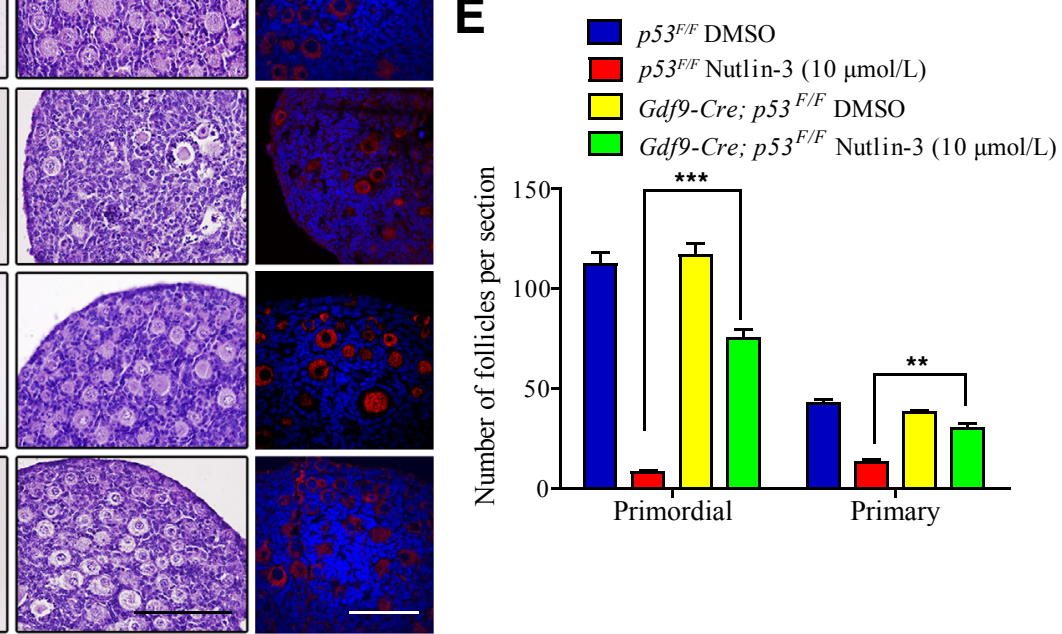

Figure 5 Nutlin-3 induced oocyte death required p53. A: H\&E-stained sections of P5 WT ovaries cultured for 4 days with either DMS0 or Nutlin-3 (2 $\mu$ mol/L, $5 \mu \mathrm{mol} / \mathrm{L}$, and $10 \mu \mathrm{mol} / \mathrm{L}$, respectively). B: Immunofluorescent staining of cleaved caspase-3 (red) in paraffin sections of P5 WT ovaries cultured for 48 hours with either DMSO or Nutlin-3 $(10 \mu \mathrm{mol} / \mathrm{L})$. C: Representative images of $\mathrm{p} 53$ immunofluorescence (green) in frozen sections of P5 WT ovaries cultured for 48 hours with either DMSO or Nutlin-3 $(10 \mu \mathrm{mol} / \mathrm{L})$. D: H\&E-stained sections of ovaries (left and middle panels) from P5 $p 53^{\mathrm{F} / F}$ and Gdff-Cre; $p 53^{\mathrm{F} / F}$ mice cultured for 4 days with either DMSO or Nutlin-3 $(10 \mu \mathrm{mol} / \mathrm{L})$. Follicles are clearly identified by immunofluorescent staining for oocyte marker DDX4 (right panel, red). E: The average number of primordial and primary follicles per section is quantified in Gdfg-Cre; $p 53^{\mathrm{F} / \mathrm{F}}$ and $p 53^{\mathrm{F} / \mathrm{F}}$ ovaries cultured with DMSO or Nutlin-3 (10 $\mu$ mol/L). Data are expressed as means \pm SEM. $n=4 \mathrm{Gdfg}$-Cre; $p 53^{F / F}$ ovaries $(\mathbf{E}) ; n=4 p 53^{F / F}$ ovaries $(\mathbf{E}) .{ }^{* *} P<0.01,{ }^{* * *} P<0.001$. Scale bars: $100 \mu \mathrm{m}(\mathbf{A}$ and $\mathbf{D}) ; 25 \mu \mathrm{m}$ (B and C). DDX4, DEAD (Asp-Glu-Ala-Asp) box polypeptide 4; DMSO, dimethyl sulfoxide; H\&E, hematoxylin and eosin; P, postnatal day; WT, wild-type.

determine the sensitivity of oocytes to 5-FU and doxorubicin treatment.

\section{Discussion}

Control of p53 activity by MDM2 and MDM4 in somatic cells is essential for embryogenesis and organogenesis to progress normally. ${ }^{41,42}$ Importantly, various stress stimuli activate $\mathrm{p} 53$ by relieving the negative regulations imposed by these factors. Thus, the negative regulations of p53 are a critical determinant for the execution of p53-mediated cellular responses. The differential role of p53 on DNA damage in mouse oocytes promotes the investigation of the control of the MDM2-p53 network in female germ cells. In this study, we demonstrated that the complete disruption of 
A

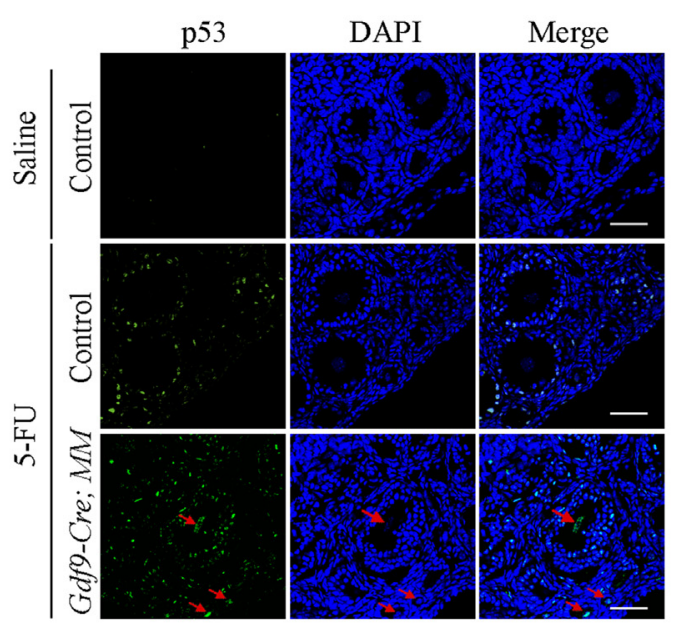

B

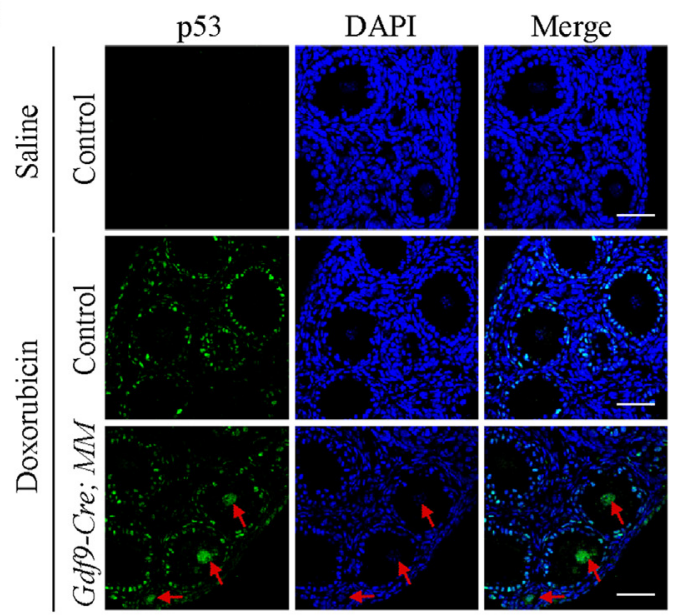

C

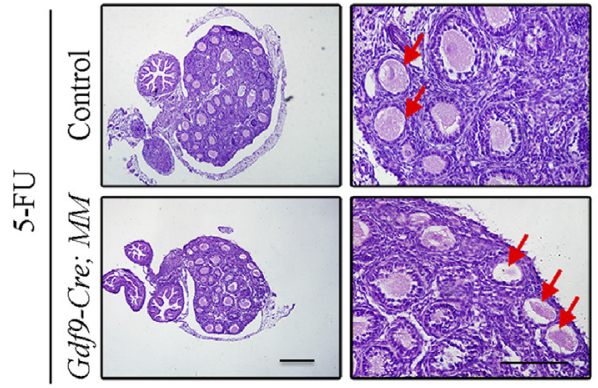

Figure 6 Treatments of 5-FU and doxorubicin induced p53 activation in oocytes of Gdfg-Cre; MM mice. A and B: Immunofluorescent staining of p53 (green) in ovarian frozen sections of P10 Gdf9-Cre; MM mice and the control littermates 24 hours after a single intraperitoneal injection of $5-\mathrm{FU}(\mathbf{A})$ or doxorubicin (B). Arrows depict positive stained nuclei. C: H\&E-stained sections of ovaries from P12 Gdf9-Cre; $M M$ mice and the control littermates 72 hours after a single intraperitoneal injection of 5-FU. Arrows depict intact oocytes in the upper panel and apoptotic oocytes in the lower panel. Scale bars: $50 \mu \mathrm{m}$ (A and B); $100 \mu \mathrm{m}($ C). 5-FU, fluorouracil; H\&E, hematoxylin and eosin; P, postnatal day. the MDM2-p53 network in mouse oocytes resulted in impeded fertility and POF phenotypes. Thus, tight regulation of p53 by MDM2 is absolutely required for oocytes survival and folliculogenesis. Further study also suggested a dose-dependent regulatory role of MDM2 and MDM4 in inhibiting p53 activation in oocytes in the presence of chemotherapeutic drugs 5-FU and doxorubicin.

Oocyte-specific deletion of $M d m 2$ resulted in a rapid loss of primordial follicles in the Gdf9-Cre; $M d m 2^{F M / F M}$ females. Because the number of primordial follicles remaining in the ovary represents the ovarian reserve that is the direct determinant of the reproductive life span, ${ }^{43}$ the early and rapid depletion of the primordial follicles due to oocyte death led to the infertility and POF phenotypes. In the subsequent study, $M d m 2$ was deleted in the growing follicles in the Zp3-Cre; $M d m 2^{F M / F M}$ mice. Although primordial follicles remained intact, there was a drastic loss of secondary and more mature follicles in these mice, leading to progressive reproductive failure and eventually the POF phenotypes. The follicular loss in this context may be due to oocyte degeneration and defective developmental progression of the follicles, eventually leading to follicular atresia. Transition from primary to secondary follicles is driven by local intraovarian autocrine and/or paracrine factors such as growth factors, cytokines, and steroids that are produced by individual component of the follicles, the oocyte, granulosa cells, and theca cells. ${ }^{33}$ The increased expression of growth inhibitory molecules due to $M d m 2$ deletion may initiate the deterioration of the oocytes and may further disrupt the communications between the oocyte and granulosa cells, resulting in the loss of follicles, especially the secondary follicles. Therefore, the Mdm2 deletion at different stages of folliculogenesis resulted in mouse phenotypes resembling human POF phenotypes with either early depletion of ovarian reserve or defects in follicular growth and maturation, respectively.

Increased nuclear accumulation of the p53 protein and the activation of p53 downstream target genes in the Mdm2-deleted oocytes were consistent with the role of MDM2 as a main negative regulator controlling p53 stability and activity. Importantly, concurrent deletions of $M d m 2$ and $p 53$ rescued the reproductive defects in the Gdf9-Cre; $M d m 2^{F M / F M}$ and Zp3-Cre; Mdm $2^{F M / F M}$ mice, further demonstrating the conserved nature of the p53MDM2 network in both somatic and germ cells. Therefore, MDM2 is actively suppressing p53 activity in the mouse oocyte for the survival and development of follicles. These results also suggested that MDM2 may not be regulating p63 and p73 as some other studies in somatic cells have suggested. ${ }^{44-47}$ Although both p63 and p73 or their orthologues have been implicated in monitoring germlines in various species, the regulation of p53 by MDM2 seemed to be highly specific and conserved in both 
somatic cells and germ cells. ${ }^{48}$ Nutlin-3 might represent an alternative to the current cytotoxic chemotherapy in particular for pediatric tumors and hematologic malignancies and is currently in a phase 1 clinical trial for the treatment of retinoblastoma. ${ }^{49}$ Our findings here suggested that measures might need to be taken to preserve the fertility of the female patients when necessary with Nutlin3 treatment.

A recent advance indicated a combinatorial role of both p63 and p53 in the response to DNA damage in the oocyte of primordial follicles because only the p63/p53 doubledeficient oocytes survived in the long term after the irradiation treatment. ${ }^{21}$ Although oocytes with $p 53$ deletion succumbed to rapid apoptosis, oocytes with p63 deletion gradually died by 7 days, ${ }^{21}$ suggesting the possibility of a relatively slow dynamics of p53 activation in oocytes on stresses. Indeed, in sharp contrast to the granulosa cells, p53 is hardly detectable in oocytes even in the presence of chemotherapeutic drugs 5-FU and doxorubicin. However, both the treatment with Nutlin-3, which directly antagonizes p53-MDM2 binding, and genetic deletion of Mdm2 in oocytes resulted in acute p53 activation and robust oocyte death, especially in primordial follicles, representing the full potential of p53 activation in oocytes. Moreover, 5-FU and doxorubicin treatment was able to promote significant p53 nuclear accumulation only in the oocytes of Gdf9-Cre; MM mice. $M d m 2$ and $M d m 4$ haploinsufficiency is known to lead to increased p53 activity in somatic cells. ${ }^{39,40}$ These results strongly suggested that the negative regulation of p53 is a key determinant of p53 activity and might be more strengthened in the oocytes versus somatic cells. Because 5-FU and doxorubicin could induce DNA damage as well as ribosome stress, both conditions of which are known to attenuate $\mathrm{p} 53-\mathrm{MDM} 2$ interactions in somatic cells ${ }^{50,51}$ via various signaling mechanisms, it is also possible that the stress-induced signaling pathways that led to the release of negative regulations on p53 might be less efficient in the oocytes. Future studies are required to address the exact mechanisms of such regulations in oocytes. Nonetheless, this study pointed to the conserved negative regulatory mechanism of p53 not only operative for the maintenance of the follicular reserve and their development but also linked to the degree of stress responses as dictated by p53 in oocytes. In addition, because p63 expression is restricted in the oocytes of small follicles, this study also revealed the effects of p53 activation in the deterioration and atresia of growing and large follicles. Given the obvious p53 activation in the granulosa cells of the large follicles in the presence of chemotherapeutic drugs, a combined effect of p53 in both oocytes and somatic cells may strongly influence the maturation of follicles and may lead to infertility under such circumstances.

Collectively, although p63 and p53 share the responsibility of monitoring germline in the presence of stresses, this study provided evidence that p53 alone is able to induce oocyte apoptosis, which is tightly and actively suppressed by its inhibitors. The tightened control of p53 activity in germ cells in the presence of stresses may explain its predominant role in regulating stress responses of somatic cells to reduce the risk of malignant transformation.

\section{Acknowledgments}

We thank Guillermina Lozano (M.D. Anderson Cancer Center, Houston, TX) for Mdm2- and Mdm4-mutant mice.

G.L. conceived the study; C.-X.Z., Q.Z., and G.L. designed the experiments and evaluated data; C.-X.Z., Q.Z., Y.-Y.X., X.-Y.H., C.X., X.-S.H., Y.Z., L.C., and G.-X.Z. performed the experiments; C.-X.Z. and G.L. wrote the manuscript.

\section{Supplemental Data}

Supplemental material for this article can be found at http://dx.doi.org/10.1016/j.ajpath.2016.09.023.

\section{References}

1. Anasti JN: Premature ovarian failure: an update. Fertil Steril 1998 , $70: 1-15$

2. Santoro N: Mechanisms of premature ovarian failure. Ann Endocrinol (Paris) 2003, 64:87-92

3. Levine A, Hu W, Feng Z: The P53 pathway: what questions remain to be explored? Cell Death Differ 2006, 13:1027-1036

4. Haupt Y, Maya R, Kazaz A, Oren M: Mdm2 promotes the rapid degradation of p53. Nature 1997, 387:296-299

5. Kubbutat MH, Jones SN, Vousden KH: Regulation of p53 stability by Mdm2. Nature 1997, 387:299-303

6. Barboza JA, Iwakuma T, Terzian T, El-Naggar AK, Lozano G: Mdm2 and Mdm4 loss regulates distinct p53 activities. Mol Cancer Res 2008, 6:947-954

7. Perry ME: The regulation of the p53-mediated stress response by MDM2 and MDM4. Cold Spring Harb Perspect Biol 2010, 2 a000968

8. el-Deiry WS, Tokino T, Velculescu VE, Levy DB, Parsons R, Trent JM, Lin D, Mercer WE, Kinzler KW, Vogelstein B: WAF1, a potential mediator of p53 tumor suppression. Cell 1993, 75:817-825

9. Nakano K, Vousden KH: PUMA, a novel proapoptotic gene, is induced by p53. Mol Cell 2001, 7:683-694

10. Oda E, Ohki R, Murasawa H, Nemoto J, Shibue T, Yamashita T, Tokino T, Taniguchi T, Tanaka N: Noxa, a BH3-only member of the Bcl-2 family and candidate mediator of p53-induced apoptosis. Science 2000, 288:1053-1058

11. Miyashita T, Reed JC: Tumor suppressor p53 is a direct transcriptional activator of the human bax gene. Cell 1995, 80:293-299

12. Hu W, Feng Z, Teresky AK, Levine AJ: p53 regulates maternal reproduction through LIF. Nature 2007, 450:721-724

13. Hu W, Feng Z, Atwal GS, Levine AJ: p53: a new player in reproduction. Cell Cycle 2008, 7:848-852

14. Derry WB, Putzke AP, Rothman JH: Caenorhabditis elegans p53: role in apoptosis, meiosis, and stress resistance. Science 2001, 294: $591-595$

15. Schumacher B, Hofmann K, Boulton S, Gartner A: The C. elegans homolog of the p53 tumor suppressor is required for DNA damageinduced apoptosis. Curr Biol 2001, 11:1722-1727 
16. Suh EK, Yang A, Kettenbach A, Bamberger C, Michaelis AH, Zhu Z, Elvin JA, Bronson RT, Crum CP, McKeon F: p63 protects the female germ line during meiotic arrest. Nature 2006, 444:624-628

17. Livera G, Petre-Lazar B, Guerquin MJ, Trautmann E, Coffigny H, Habert R: p63 null mutation protects mouse oocytes from radioinduced apoptosis. Reproduction 2008, 135:3-12

18. Guerquin MJ, Duquenne C, Coffigny $\mathrm{H}$, Rouiller-Fabre $\mathrm{V}$, Lambrot R, Bakalska M, Frydman R, Habert R, Livera G: Sexspecific differences in fetal germ cell apoptosis induced by ionizing radiation. Hum Reprod 2009, 24:670-678

19. Gonfloni S, Di Tella L, Caldarola S, Cannata SM, Klinger FG, Di Bartolomeo C, Mattei M, Candi E, De Felici M, Melino G, Cesareni G: Inhibition of the c-Abl-TAp63 pathway protects mouse oocytes from chemotherapy-induced death. Nat Med 2009, 15:1179-1185

20. Kim SY, Cordeiro MH, Serna VA, Ebbert K, Butler LM, Sinha S, Mills AA, Woodruff TK, Kurita T: Rescue of platinum-damaged oocytes from programmed cell death through inactivation of the p53 family signaling network. Cell Death Differ 2013, 20:987-997

21. Bolcun-Filas E, Rinaldi VD, White ME, Schimenti JC: Reversal of female infertility by Chk2 ablation reveals the oocyte DNA damage checkpoint pathway. Science 2014, 343:533-536

22. Lan ZJ, Xu X, Cooney AJ: Differential oocyte-specific expression of Cre recombinase activity in GDF-9-iCre, Zp3cre, and Msx2Cre transgenic mice. Biol Reprod 2004, 71:1469-1474

23. Lewandoski M, Wassarman KM, Martin GR: Zp3-cre, a transgenic mouse line for the activation or inactivation of loxP-flanked target genes specifically in the female germ line. Curr Biol 1997, 7:148-151

24. Grier JD, Yan W, Lozano G: Conditional allele of mdm2 which encodes a p53 inhibitor. Genesis 2002, 32:145-147

25. Xiong S, Van Pelt CS, Elizondo-Fraire AC, Liu G, Lozano G: Synergistic roles of Mdm2 and Mdm4 for p53 inhibition in central nervous system development. Proc Natl Acad Sci U S A 2006, $103: 3226$

26. Marino S, Vooijs M, van Der Gulden H, Jonkers J, Berns A: Induction of medulloblastomas in p53-null mutant mice by somatic inactivation of $\mathrm{Rb}$ in the external granular layer cells of the cerebellum. Genes Dev 2000, 14:994-1004

27. Byers SL, Wiles MV, Dunn SL, Taft RA: Mouse estrous cycle identification tool and images. PLoS One 2012, 7:e35538

28. Tilly JL: Ovarian follicle counts-not as simple as 1, 2, 3. Reprod Biol Endocrinol 2003, 1:11

29. Goswami D, Conway GS: Premature ovarian failure. Horm Res 2007, 68:196-202

30. Broekmans FJ, Knauff EA, te Velde ER, Macklon NS, Fauser BC: Female reproductive ageing: current knowledge and future trends. Trends Endocrinol Metab 2007, 18:58-65

31. Hansen KR, Knowlton NS, Thyer AC, Charleston JS, Soules MR, Klein NA: A new model of reproductive aging: the decline in ovarian non-growing follicle number from birth to menopause. Hum Reprod 2008, 23:699-708

32. de Vries WN, Binns LT, Fancher KS, Dean J, Moore R, Kemler R, Knowles BB: Expression of Cre recombinase in mouse oocytes: a means to study maternal effect genes. Genesis 2000, 26:110-112

33. Kol S, Adashi EY: Intraovarian factors regulating ovarian function. Curr Opin Obstet Gynecol 1995, 7:209-213

34. Kidder GM, Mhawi AA: Gap junctions and ovarian folliculogenesis. Reproduction 2002, 123:613-620
35. Li J, Ye Y, Zhang R, Zhang L, Hu X, Han D, Chen J, He X, Wang G, Yang X, Wang L: Robo1/2 regulate follicle atresia through manipulating granulosa cell apoptosis in mice. Sci Rep 2015, 5:9720

36. Shangary S, Wang S: Small-molecule inhibitors of the MDM2-p53 protein-protein interaction to reactivate p53 function: a novel approach for cancer therapy. Annu Rev Pharmacol Toxicol 2009, 49: 223-241

37. Vassilev LT, Vu BT, Graves B, Carvajal D, Podlaski F, Filipovic Z, Kong N, Kammlott U, Lukacs C, Klein C, Fotouhi N, Liu EA: In vivo activation of the p53 pathway by small-molecule antagonists of MDM2. Science 2004, 303:844-848

38. Tovar C, Rosinski J, Filipovic Z, Higgins B, Kolinsky K, Hilton H, Zhao X, Vu BT, Qing W, Packman K, Myklebost O, Heimbrook DC, Vassilev LT: Small-molecule MDM2 antagonists reveal aberrant p53 signaling in cancer: implications for therapy. Proc Natl Acad Sci U S A 2006, 103:1888-1893

39. Terzian T, Wang Y, Van Pelt CS, Box NF, Travis EL, Lozano G: Haploinsufficiency of $\mathrm{Mdm} 2$ and $\mathrm{Mdm} 4$ in tumorigenesis and development. Mol Cell Biol 2007, 27:5479-5485

40. Zhang Q, He X, Chen L, Zhang C, Gao X, Yang Z, Liu G: Synergistic regulation of $\mathrm{p} 53$ by $\mathrm{Mdm} 2$ and $\mathrm{Mdm} 4$ is critical in cardiac endocardial cushion morphogenesis during heart development. J Pathol 2012, 228:416-428

41. Chavez-Reyes A, Parant JM, Amelse LL, de Oca Luna RM, Korsmeyer SJ, Lozano G: Switching mechanisms of cell death in mdm2- and mdm4-null mice by deletion of p53 downstream targets. Cancer Res 2003, 63:8664-8669

42. de Rozieres S, Maya R, Oren M, Lozano G: The loss of mdm2 induces p53-mediated apoptosis. Oncogene 2000, 19:1691-1697

43. Zhang H, Zheng W, Shen Y, Adhikari D, Ueno H, Liu K: Experimental evidence showing that no mitotically active female germline progenitors exist in postnatal mouse ovaries. Proc Natl Acad Sci U S A 2012, 109:12580-12585

44. Zeng X, Chen L, Jost CA, Maya R, Keller D, Wang X, Kaelin WG Jr, Oren M, Chen J, Lu H: MDM2 suppresses p73 function without promoting p73 degradation. Mol Cell Biol 1999, 19:3257-3266

45. Rossi M, De Laurenzi V, Munarriz E, Green DR, Liu YC, Vousden KH, Cesareni G, Melino G: The ubiquitin-protein ligase Itch regulates p73 stability. EMBO J 2005, 24:836-848

46. Calabro V, Mansueto G, Parisi T, Vivo M, Calogero RA, La Mantia G: The human MDM2 oncoprotein increases the transcriptional activity and the protein level of the p53 homolog p63. J Biol Chem 2002, 277:2674-2681

47. Kadakia M, Slader C, Berberich SJ: Regulation of p63 function by Mdm2 and MdmX. DNA Cell Biol 2001, 20:321-330

48. Lane DP, Cheok CF, Brown C, Madhumalar A, Ghadessy FJ, Verma C: Mdm2 and p53 are highly conserved from placozoans to man. Cell Cycle 2010, 9:540-547

49. Secchiero P, Bosco R, Celeghini C, Zauli G: Recent advances in the therapeutic perspectives of Nutlin-3. Curr Pharm Des 2011, 17: $569-577$

50. Rubbi CP, Milner J: Disruption of the nucleolus mediates stabilization of p53 in response to DNA damage and other stresses. EMBO J 2003, 22:6068-6077

51. Sun XX, Dai M-S, Lu H: 5-fluorouracil activation of p53 involves an MDM2-ribosomal protein interaction. J Biol Chem 2007, 282: 8052-8059 